\section{Reanalysis of data from the Million Women Study}

I was very pleased to see the article by Shapiro and colleagues ${ }^{1}$ that was published online in the Journal of Family Planning and Reproductive Health Care in February 2012 (and that appears in print in this issue) but dismayed to see the emotional response from epidemiologists. ${ }^{2}$ This reanalysis of data from the so-called 'Million Women' study ${ }^{3}$ raises important clinical concerns about the original strongly stated conclusions. I think it is essential that we see continuing debate about these complex epidemiological studies, where results are open to different interpretations.

Putting emotions aside, there are some problems with the original analysis of the Million Women Study (MWS). This type of study cannot make allowances for every possible bias, and as we are all aware 'big is not necessarily better' when biases are present. The statistically significant differences seen in the MWS are still very small, and potential biases could considerably change the final statistics.

It is the traditional scientific way to have debate about the findings of controversial studies, and, to me, it seems appropriate that the epidemiologists should set aside emotion and address the legitimate questions and criticisms of other scientists in the original journal to which the article was submitted.

The epidemiologists have managed to raise fear among women in the general community about use of hormone replacement preparations, yet these therapies have an enormous impact on many aspects of well-being, such that the benefit-risk ratio for most individual women is very positive. I would really like to show the epidemiologists I know (who do not see any patients) the dramatic impact that hormone replacement therapy (HRT) can have on the quality of the lives of many menopausal women.

We should not forget that the much vaunted and highly criticised Women's Health Initiative study showed a significant reduction in risk of breast cancer for women using estrogen-alone HRT. ${ }^{4}$ I do not hear the epidemiologists trumpeting this!

Everything we do in this life carries risk. Please can we look realistically at what are the many potential benefits of HRT and put them in perspective with individual risk. Let the debate continue without emotion!

\section{Ian S Fraser, MD, FRANZCOG}

Professor in Reproductive Medicine, Queen Elizabeth II Research Institute for Mothers and Infants, University of Sydney, Sydney, New South Wales, Australia; ian.fraser@sydney.edu.au
Competing interests None.

J Fam Plann Reprod Health Care 2012;38:136. doi:10.1136/jprhc-2012-100332

\section{References}

1 Shapiro S, Farmer RD, Stevenson JC, et al. Does hormone replacement therapy cause breast cancer? An application of causal principles to three studies: Part 4. The Million Women Study. J Fam Plann Reprod Health Care 2012;38:102-109.

2 Kmietowicz Z. Articles disputing link between HRT and breast cancer are "ridiculous". BMJ 2012;344:e513.

3 Million Women Study Collaborators. Breast cancer and hormone replacement therapy in the Million Women Study. Lancet 2003;362:419-427.

4 The Women's Health Initiative Steering Committee. Effects of conjugated equine estrogen in postmenopausal women with hysterectomy: the Women's Health Initiative randomized controlled trial. JAMA 2004;291:1701-1712. 